\title{
A sulfhydryl azobenzene modificatied polyaniline/silver electrode and its photoswitching electrochemical performance
}

Changguo Xue ${ }^{*}, \dagger$, Shiqin Li ${ }^{\dagger}$, Yu Tang $\dagger$, Cunbin An $\ddagger$, Song Liu ${ }^{\dagger}$ and Yanhua Teng ${ }^{\dagger}$

$†$ School of Material science and Engineering, Anhui University of Science and Technology, Anhui, Huainan 232001, China.

* Beijing National Laboratory for Molecular Sciences, State Key Laboratory of Polymer Physics and Chemistry, Institute of Chemistry, Chinese Academy of Sciences, Beijing 100190, China. 


\section{Results}

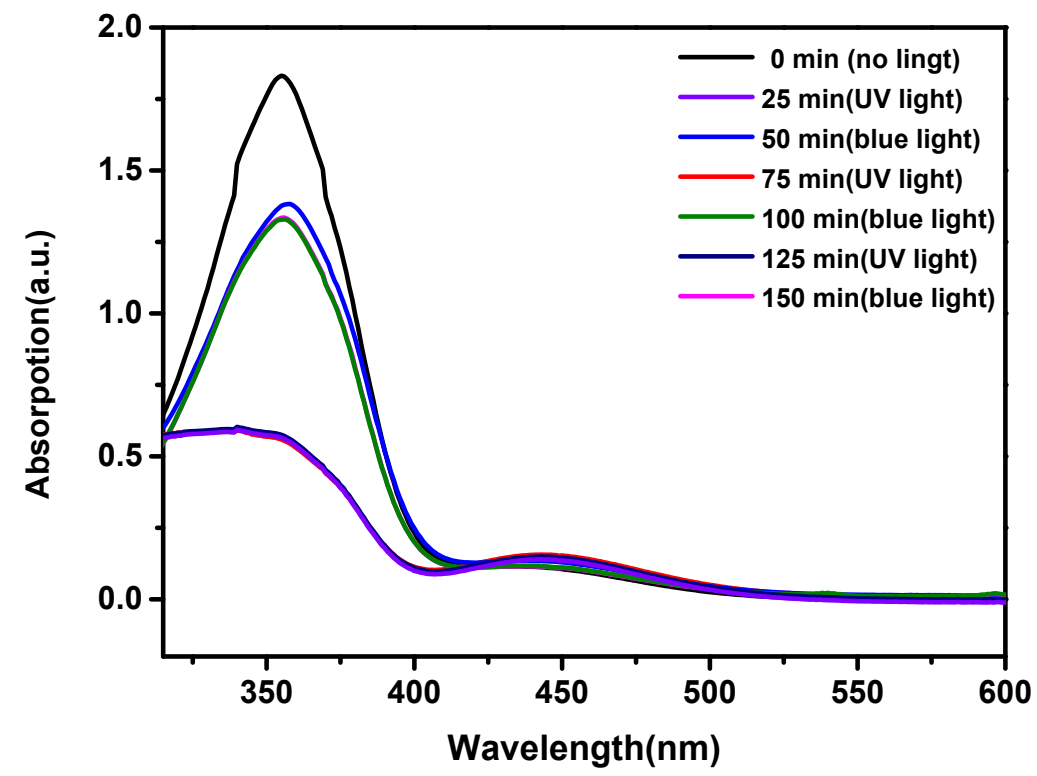

Figure S1. The changes in the absorption band of Azo molecules at $355 \mathrm{~nm}$ upon alternating irradiation by UV and blue light.

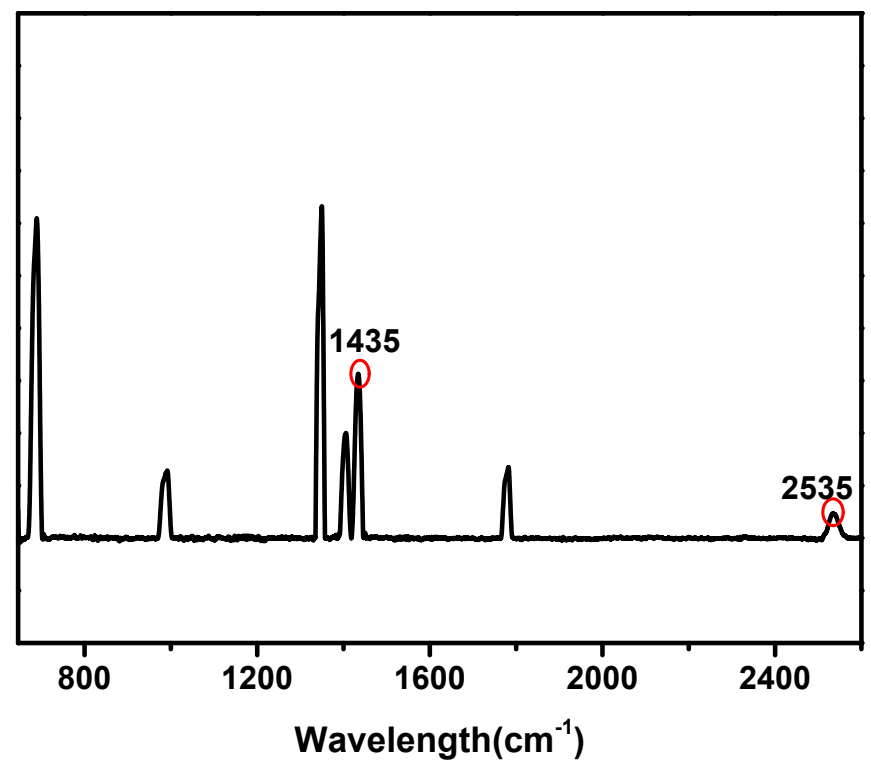

Figure S2. Raman spectra of Azo 

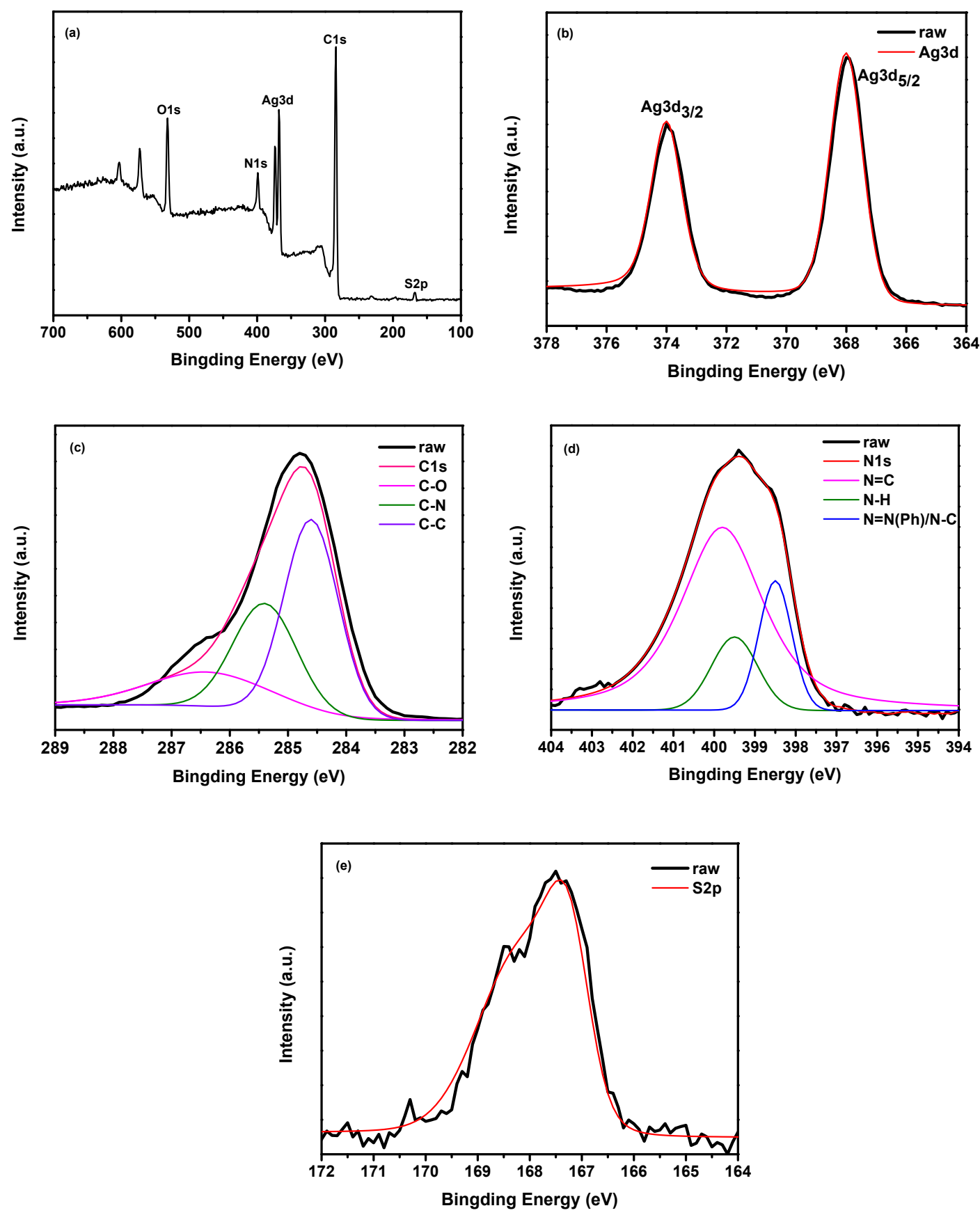

Figure S3. XPS results of (a) PANI/Ag/Azo, (b)Ag3d, (c)C1s, (d)N1s, (e)S2p 


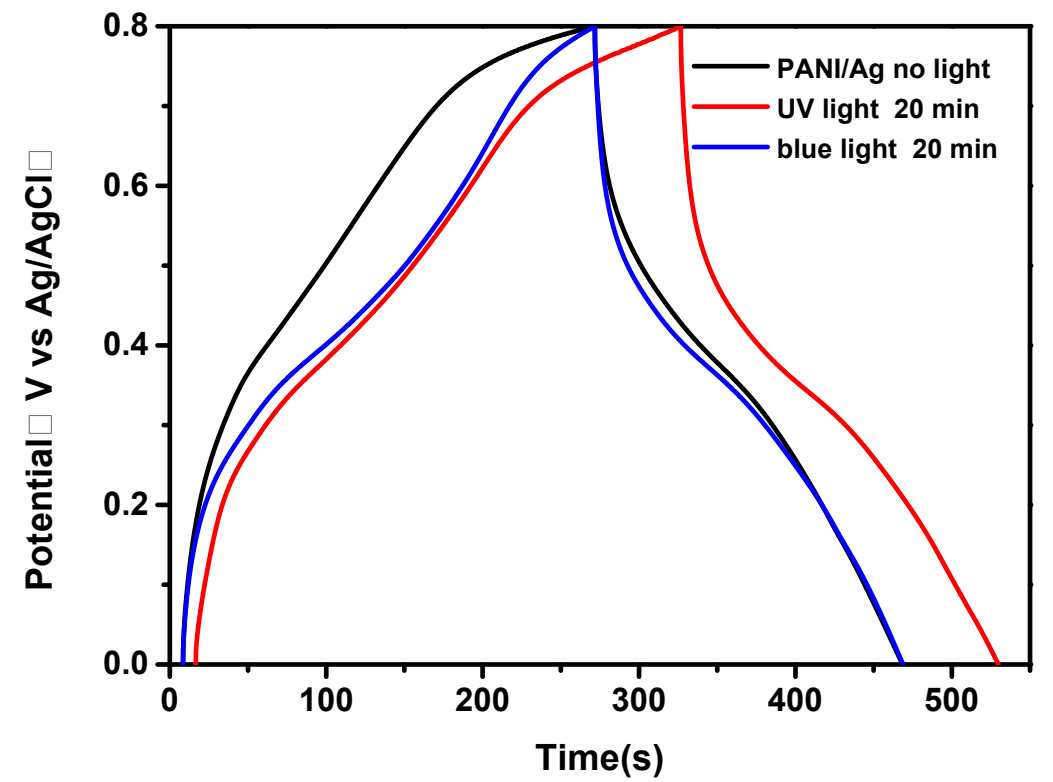

Figure S4. GCD curves of PANI/Ag after UV light and blue light irradiation.

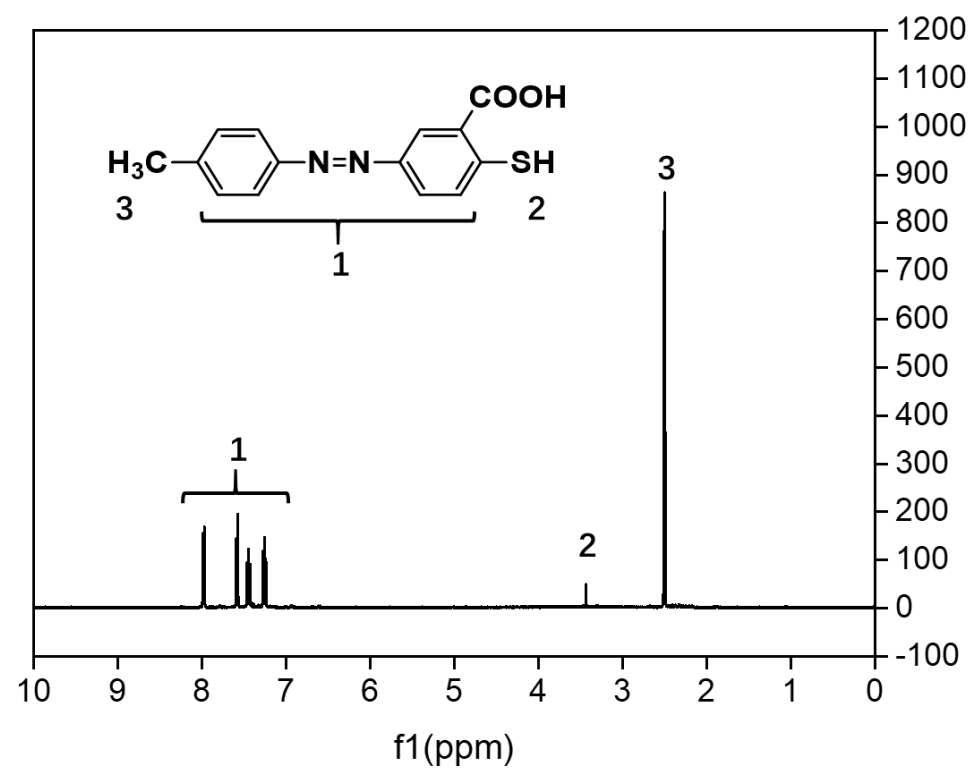

Figure S5. ${ }^{1} \mathrm{HNMR}$ spectra of Azo in dimethyl sulfoxide ( DMSO ) . 